\title{
In conflict with ourselves? An investigation of heuristic and analytic processes in decision making
}

\author{
Carissa Bonner and Ben R. Newell \\ University of New South Wales, Sydney, New South Wales, Australia
}

\begin{abstract}
Many theorists propose two types of processing: heuristic and analytic. In conflict tasks, in which these processing types lead to opposing responses, giving the analytic response may require both detection and resolution of the conflict. The ratio bias task, in which people tend to treat larger numbered ratios (e.g., 20/100) as indicating a higher likelihood of winning than do equivalent smaller numbered ratios (e.g., 2/10), is considered to induce such a conflict. Experiment 1 showed response time differences associated with conflict detection, resolution, and the amount of conflict induced. The conflict detection and resolution effects were replicated in Experiment 2 and were not affected by decreasing the influence of the heuristic response or decreasing the capacity to make the analytic response. The results are consistent with dual-process accounts, but a single-process account in which quantitative, rather than qualitative, differences in processing are assumed fares equally well in explaining the data.
\end{abstract}

Dual-process theories propose two distinct types of reasoning: heuristic processing, which is fast and automatic and does not tax cognitive resources, and analytic processing, which is slow, rule based, and dependent on working memory capacity (Evans, 2006). This basic heuristic/analytic distinction is common across many different theories but is described in a variety of ways, such as experiential/rational (Denes-Raj \& Epstein, 1994; Kirkpatrick \& Epstein, 1992), associative/rule-based (Sloman, 1996), or simply System 1/System 2 (Evans, 2008; Stanovich \& West, 2000). A keenly debated but unresolved issue for dual-process theories is how processes cooperate, compete, or, more generally, exchange information in order to determine a given response (Evans, 2007, 2008; Mitchell, DeHouwer, \& Lovibond, 2009; Newell, 2009). An appealing recent line of research has suggested that taking a closer look at the proposed conflict between heuristic and analytic processing can shed light on how this interaction occurs (e.g., De Neys \& Glumicic, 2008). The present study extended this line of research by examining a simple task that has often been interpreted as creating a conflict between heuristic and analytic responses: the ratio bias task. The research is novel in that it generalizes empirical tests of the processes underlying the proposed conflict to a new reasoning task and uses new methods of measurement in that task (processing speed). Furthermore, although distinguishing between dual-process and single-process models was not a direct aim of the research, the data highlight that patterns of responding used as evidence of conflict between processes can also be interpreted as reflecting a single process (e.g., Hammond, 1996; Osman, 2004).

\section{Conflict Between Types of Reasoning}

Whether focused on personality differences (e.g., DenesRaj \& Epstein, 1994), cognitive ability (e.g., Stanovich \& West, 1998), or the specific operation of different systems (e.g., Sloman, 1996), dual-process researchers have tested their predictions by using the idea that two types of reasoning can conflict in certain situations. For example, in the classic base rate neglect problems (Kahneman \& Tversky, 1973), participants read about a sample of people in which most belong to one category (e.g., 995 women) but some belong to another (e.g., 5 men). When the description of a randomly drawn person from the sample is consistent with the stereotype of the less likely category (men), people often ignore the small base rate $(5 / 1,000)$ and say that the person is most likely to be from this category. To give the normatively correct response (i.e., the statistically most likely category: women), instead of the compelling heuristic response, all of the available information must be utilized, presumably through the use of analytic processing (Gigerenzer, Hell, \& Blank, 1988). Since the two types of reasoning lead to different responses and people have been shown to give either response, heuristic and analytic processing have been conceptualized as being in conflict with each other. As Denes-Raj and Epstein (1994, p. 819) anecdotally described, people seem to "know" the correct option but still "feel" like they should choose the other,

B. R. Newell, ben.newell@unsw.edu.au 
and this conflict can lead people to "behave against their better judgment."

\section{Conflict Detection and Resolution}

De Neys and colleagues have investigated the nature of this apparent conflict in detail, finding evidence for three proposed aspects of the reasoning process: conflict monitoring, conflict detection, and conflict resolution through inhibition (De Neys, 2006a, 2006b; De Neys \& Glumicic, 2008; De Neys, Vartanian, \& Goel, 2008).

In a series of experiments, De Neys and Glumicic (2008) tested whether heuristic and analytic processing occur in parallel, so that output is constantly monitored for potential conflict (Denes-Raj \& Epstein, 1994; Sloman, 1996), or whether conflict monitoring is ineffective because heuristic processing actually precedes analytic processing (Evans, 2006, 2007; Kahneman \& Frederick, 2002). They gave participants base rate neglect problems and measured their attention to and knowledge of the base rate in a variety of ways. Although most participants did not explicitly mention the base rate when explaining their responses, even those who gave the heuristic response were shown to have specifically reviewed the base rate information and were able to recall the base rate when asked. On the basis of these findings, the authors argued that nonoptimal heuristic responses, such as choosing the normatively less likely response, are caused not by a lack of conflict detection, but by a failure to resolve the conflict (presumably, through inhibition of the heuristic response) after the conflict has been detected. This pattern is consistent with parallel models, which assume that there is effective, rather than "lax," monitoring of the output of both types of processing.

This idea of conflict detection and resolution is further supported by response time differences (De Neys, 2006a; De Neys \& Glumicic, 2008) and neuroimaging evidence (De Neys et al., 2008). For conflict detection, problems that lead to a conflict between heuristic and analytic responses take longer to answer than problems without such a conflict, suggesting that the mere presence of a conflict slows processing. Such problems also activate the brain region believed to be associated with conflict detection (the anterior cingulate cortex). These findings hold even when respondents give the heuristically cued response, indicating once again that failure to resolve the conflict seems to occur after the conflict has been detected. For conflict resolution, participants with high accuracy on conflict problems, who presumably take the extra step of inhibiting the heuristic response, take longer to respond than do participants with low accuracy, who give the heuristic response. Normatively correct responses have also been shown to activate the brain region believed to be involved in response inhibition (the right lateral prefrontal cortex).

\section{Heuristic, Analytic, and Normative Responding}

De Neys and Glumicic (2008) interpreted a base rate response as indicating the involvement of the analytic process and a representative, or stereotype-based, response as indicating heuristic processing. They also described the base rate response as normatively correct and the representative response as erroneous (although see their note 3 ). However, these are not the only possible interpretations of their results. As Evans (2007) pointed out, nothing in the definition of heuristic and analytic processes implies normativity. The definitions describe the nature of processing (fast, slow), the representations used by the processes (rules, associations), and the mode of operation (sequential, parallel). A fast, intuitive response can often be correct (e.g., those based on recognition; Goldstein \& Gigerenzer, 2002), and slow, deliberative responding can still result in "poor" decision making (Wilson \& Schooler, 1991). Thus, a base rate response, just because it is (arguably) normatively correct, does not necessarily imply the use of analytic processing. Indeed, there is evidence to suggest that people find it very difficult to engage in the Bayesian reasoning required to fully understand the tasks used by De Neys and Glumicic (in the absence of appropriate problem formats; see, e.g., Sloman, Over, Slovak, \& Stibel, 2003). Such reasoning requires integration of the stereotype and base rate information, rather than relying on one or the other (Gigerenzer et al., 1988).

Since De Neys and Glumicic (2008) used a binary choice measure rather than probability estimates, it is possible that the base rate responses that they observed were not the product of rule-based analytic processing but of a simple choice between using one of two separate pieces of information. It could, therefore, be suggested that they created a conflict between two types of heuristic responding (use only the stereotype information or use only the base rate information), rather than heuristic and analytic (use only the stereotype information or integrate this information with the base rate).

This uncertainty about the nature of the processing underlying a base rate response has been addressed in more recent research (Franssens \& De Neys, 2009), but the complexity of integrating stereotype and base rate information could be avoided altogether by using a simpler conflict task. A simpler task would have the additional advantage of reducing response time length, since even those who give the putatively heuristic response in the research above had quite long response times $(M>10 \mathrm{sec}$ for all groups and tasks). If the heuristic response is automatically cued, as dual-system theorists generally claim (Evans, 2008), it should be possible to find response time differences on a much shorter time scale, to more closely reflect actual processing speed. The ratio bias paradigm developed by Epstein and colleagues (Denes-Raj \& Epstein, 1994; Kirkpatrick \& Epstein, 1992) provides a good basis for such a conflict task. As is explained in detail below, the heuristic response is based on a simple external cue, the analytic response requires rule-based integration of this cue with additional information, and the relative simplicity of the task means that either response can be given quickly.

\section{The Ratio Bias Paradigm}

Research on the ratio bias has been used to support one particular dual-process theory, cognitive-experiential self theory (CEST) (Alonso \& Fernández-Berrocal, 2003; Denes-Raj \& Epstein, 1994; Epstein \& Pacini, 20002001; Kirkpatrick \& Epstein, 1992). 
In the basic ratio bias task, participants are given a choice of two trays containing red and white jelly beans, and there are always more white than red jelly beans. There is one small tray (e.g., 2 red out of a total of 10 jelly beans) and one large tray (e.g., 20 red out of a total of 100 jelly beans). The participants must choose one of the trays, from which 1 jelly bean will be randomly drawn by the experimenter. If the selected bean is red, the participants win money; if it is white, they win nothing.

The ratio bias is the tendency to treat larger numbered ratios (e.g., 20/100) as indicating a higher likelihood of winning than equivalent smaller numbered ratios (e.g., 2/10) (Kirkpatrick \& Epstein, 1992). This tendency is strong enough to lead people to make "irrational" choices, such as choosing $9 / 100(9 \%)$ over a $1 / 10(10 \%)$ chance of winning (Denes-Raj \& Epstein, 1994). The less optimal the heuristic choice is, the easier it appears to be to choose rationally, since people are less likely to choose $7 / 100(7 \%)$ than $9 / 100(9 \%)$ when compared with a $1 / 10$ $(10 \%)$ alternative. Nevertheless, some participants have been shown to choose options with only half the probability of winning. Dale, Rudski, Schwarz, and Smith (2007) further demonstrated that the ratio bias cannot be attributed to general irrationality or error, since nonoptimal responses have been found much more frequently when the small tray is the optimal choice (e.g., 9/100 vs. $1 / 10$ ) than when the large tray is the optimal choice (e.g., $11 / 100$ vs. $1 / 10)$.

According to CEST, such nonoptimal responses are found because the experiential (heuristic) system focuses on the numerator of the ratio (e.g., 9 vs. 1) instead of the overall probability (e.g., $9 \%$ vs. $10 \%$ ), which requires the input of the rational (analytic) system. The size of the monetary incentive may increase the motivation to give the rational response but does not eliminate the ratio bias entirely (Dale et al., 2007).

The ratio bias effect has also been demonstrated in more hypothetical situations, such as preferences for job applications (Alonso \& Fernández-Berrocal, 2003) and perceptions of health-related risk (Bonner \& Newell, 2008; Pinto-Prades, Martinez-Perez, \& Abellán-Perpiñán, 2006; Yamagishi, 1997). Since making a ratio bias response in the jelly bean task is more obviously an "irrational" choice, using a hypothetical situation requires some instructional manipulation. Epstein and Pacini (2000-2001) found that it was necessary to reduce participants' desire to "appear" rational by asking participants what they thought others would do, rather than what they themselves would do. This was found to help equate the outcome of behavioral and hypothetical situations. The present research drew on this finding by describing the jelly bean task and asking participants what they thought other people would do, rather than using a behavioral situation with a monetary incentive.

\section{Task Features}

There are two notable differences between the base rate neglect problems used in De Neys's conflict research, and the ratio bias task developed through Epstein's work.
First, there is a difference in the source of the heuristic response. In the ratio bias task, the heuristic response is cued by an external source, the numerator of the ratios, which can be made more or less salient through the use of different presentation formats (Rudski \& Volksdorf, 2002). In base rate neglect problems, the heuristic response is cued by an internal source, group stereotypes based on prior beliefs, which may differ in salience, depending on the individual.

Second, there is a difference in how participants use analytic processing to come to the optimal response. In the ratio bias task, both heuristic and analytic processing of the problem rely on the same external source of information. The difference between the types of processing is in how much of this information is utilized (just the numerator, or integrating this with the denominator). Analytic processing to reach the optimal response is based on a single identifiable rule: Calculate the percentage, using the numerator and denominator of each ratio, and choose the higher percentage. In contrast, analytic processing in the base rate neglect task requires that participants integrate information from internal and external sources. Gigerenzer et al. (1988) used self-reported strategies and compared probability judgments with Bayesian performance to show that this might be done in a variety of ways. This means that different participants who give the optimal response can use different strategies to reach the same answer.

In addition, previous research suggests that the ratio bias task is well suited for investigating conflict between proposed heuristic and analytic processes. Increasing the salience of the heuristic cue (i.e., the numerator of the ratio) by using a visual presentation format has been shown to decrease optimal responding (Rudski \& Volksdorf, 2002), providing support for the assumption that a nonoptimal response is based on this simple cue, rather than on all of the available information. Encouraging participants to respond in a "logical" manner has been shown to increase optimal responding (Epstein \& Pacini, 2000-2001), supporting the idea that an optimal response is the result of the available information's being fully understood and integrated in a conscious rule-based process.

\section{EXPERIMENT 1}

\section{Aim and Hypotheses}

The aim of Experiment 1 was to test hypotheses relating to the proposed concepts of conflict detection, conflict resolution, and the amount of conflict between types of processing. ${ }^{1}$

First, according to the concept of conflict detection, conflict trials (on which a different response is cued by heuristic and analytic processing; e.g., 2/10 [analytic response] vs. 19/100 [heuristic response]) will be longer than harmony trials (where the same response is cued by heuristic and analytic processing; e.g., $2 / 10$ vs. 21/100 [heuristic and analytic response]). This slowing could be the result of time taken to detect the conflict; alternatively, it might be due to participants' initiating resolution of the conflict, regardless of whether they complete this process 
to give the analytic response. Thus, it may not be the detection per se that increases latencies (this could in fact occur quickly) but the consequences of that detection.

Second, if the analytic response depends on resolution of the detected conflict and the heuristic response results from a failure to complete this process, we would expect those who give more analytic responses to be slower, on average, than those who give more heuristic responses. Therefore, participants who are given "permission" to go with their initial heuristic response (i.e., those given "what would MOST people do?" instructions) will be faster than those who are motivated to resolve the conflict (i.e., those given "what would COMPLETELY LOGICAL people do?" instructions).

Finally, previous research has indicated that the optimal response is less likely to be chosen when the quantitative difference between heuristic and analytic responses is small (e.g., $2 / 10$ vs. $19 / 100=1 \%$ ) than when it is large (e.g., $2 / 10$ vs. $11 / 100=9 \%)($ Denes-Raj \& Epstein, 1994). This quantitative difference could be thought of as a proxy for the amount of conflict that two competing ratios might create. How might this affect response times? One possibility is that when there is a small quantitative difference between the heuristic and analytic responses, the conflict resolution process will be more difficult to complete, leading to longer response times for small-difference trials. An alternative possibility is that the conflict would be harder to detect in the first place, leading to shorter response times for small-difference trials, because fewer people will initiate the conflict resolution process.

\section{Method}

Participants. Fifty-one undergraduate students $(66 \%$ female, $34 \%$ male) with a mean age of 20.3 years $(S D=0.540)$ participated, in return for credit in a first-year psychology course at the University of New South Wales.

Design. The experiment was a $2 \times(2 \times 3)$ design. The betweensubjects variable was the instructions: What would most people do (most people condition) or what would completely logical people do (logical people condition). The two within-subjects variables were the direction and size of the difference between ratios on each trial. Direction refers to which tray had the higher percentage of red jelly beans: the small tray (conflict trials) or the large tray (harmony trials). Size refers to the percentage difference between ratios: $1 \%-3 \%$ (small), $4 \%-6 \%$ (medium), or 7\%-9\% (large).

Materials. The ratio pairs were developed on the basis of percentage ranges used in previous research. The small tray contained 1,2 , or 3 red jelly beans out of a total 10 jelly beans (i.e., $10 \%-30 \%$ ). The large tray had a total of 100 jelly beans, and the proportion of red jelly beans differed from the small tray by a range of $-9 \%$ to $+9 \%$. The participants saw each combination of ratios only once. This resulted in 57 trials altogether: 27 conflict trials (e.g., $2 / 10$ vs. 19/100), 27 harmony trials (e.g., 2/10 vs. 21/100), and 3 equal trials (e.g., $2 / 10$ vs. 20/100).

Procedure. The participants completed the experiment on a computer. A typical jelly bean experiment was described to them, along with the phrase "the interesting thing about this experiment is that some participants show a distinct preference for one tray or the other." This phrase was used by Epstein and Pacini (2000-2001, p. 203) in a similar hypothetical scenario, in order to "make the participants feel that it was acceptable" to indicate that others might have an irrational tray preference.

The participants were then told that their task was to choose the tray that most or completely logical people select in the described jelly bean experiment. They then completed four practice trials to get used to the left and right response keys, on which they were instructed to choose the left or right tray. The tray to be selected contained either 10 or 100 white jelly beans at random, with no red jelly beans. No ratio information was displayed above the trays.

After the practice trials, the 57 experiment trials were completed in randomized order. A brief summary of the introduction was visible throughout these trials. On each trial, the question "Which tray do you think MOST/COMPLETELY LOGICAL people choose?" appeared $1 \mathrm{sec}$ before pictures of the small tray ( 10 jelly beans) and the large tray (100 jelly beans) were shown simultaneously. The numerical ratio of red jelly beans to total jelly beans (e.g., 2/10) was also shown above each tray picture. The position (left or right) of the small and large trays was randomized on each trial. The participants indicated their tray choice using the left or right response key. After their response, there was an intertrial delay of $2 \mathrm{sec}$ before the next trial began.

\section{Results}

Table 1 displays the mean proportion of nonoptimal responses and mean response time in each condition (across conflict and harmony trials). One participant was excluded from the analysis after making preemptive responses $(<200 \mathrm{msec})$ on $98 \%$ of the trials. For the remaining participants, preemptive responses $(<200 \mathrm{msec})$ were excluded, which affected $2 \%$ of the data. For the response time analysis, outliers $(>2.5 \mathrm{SDs}$ from the mean) were replaced with the cutoff value. This was done separately for conflict and harmony trials for each participant and affected $5 \%$ of the data.

Nonoptimal choice. The equal trials (i.e., those on which the small and large trays contained equal proportions of red jelly beans) were used to test whether a basic ratio bias effect (i.e., preference for the large tray) could be found within the multiple-trial design. The large tray was chosen $61.7 \%(S E=5.7 \%)$ of the time, on average, which was significantly different from the test value of $50 \%[t(49)=2.040, p=.047]$. This appeared to be driven more by the most people group $(M=72.0 \%, S E=7.4 \%)$ than by the logical people group $(M=51.3 \%, S E=$ $8.4 \%$ ), although this difference only approached significance $[F(1,48)=3.426, p=.070]$.

A $2 \times(2 \times 3)$ repeated measures ANOVA was then conducted on the proportion of nonoptimal responses, based on the 54 conflict and harmony trials (i.e., excluding the 3 equal trials). The variables were instructions (most

Table 1

Mean Percentages of Nonoptimal Choices and Mean Response Times in Experiment 1

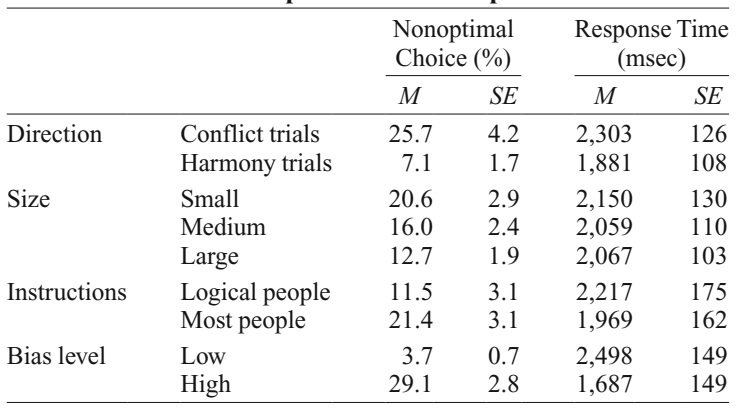




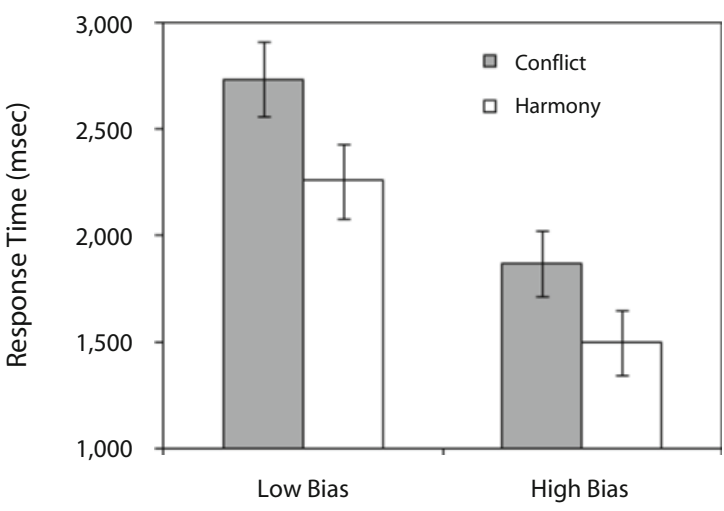

Bias Level

Figure 1. Main effects of bias level (low $>$ high) and direction (conflict $>$ harmony), collapsed across size. Bias level refers to a median split of participants based on the proportion of ratio bias responses they made (i.e., nonoptimal responses on conflict trials). Direction refers to which tray had the larger proportion of red jelly beans, the small tray (conflict trials) or the large tray (harmony trials). Size refers to the difference between the proportions of red jelly beans in the small and large trays on a given trial: small $(1 \%-3 \%)$, medium $(4 \%-6 \%)$, or large $(7 \%-9 \%)$. Error bars are one standard error of the mean.

people or logical people), direction (conflict or harmony), and size (small, medium, or large). A nonoptimal response was defined as choosing the tray with the smaller percentage chance of winning. There were three significant main effects: instructions, direction, and size. For instructions $[F(1,48)=4.967, p=.031]$, the most people group $(M=$ $21.4 \%, S E=3.1 \%$ ) was more likely to make nonoptimal responses than was the logical people group $(M=11.5 \%$, $S E=3.1 \%)$. For direction $[F(1,48)=16.342, p<.001]$, more nonoptimal responses were made on conflict trials $(M=25.7 \%, S E=4.2 \%)$ than on harmony trials $(M=$ $7.1 \%, S E=1.7 \%$ ), indicating a ratio bias effect. For size $[F(2,47)=7.366, p=.002]$, the proportion of nonoptimal responses decreased as the difference between the ratios increased, with a significant difference between small $(M=20.6 \%, S E=2.9 \%)$ and large $(M=12.7 \%, S E=$ $1.9 \%)$

Response time. Although there was an overall difference between the nonoptimal responses in the most people and logical people conditions, the proportion of ratio bias responses (i.e., nonoptimal responses in conflict trials) was higher than expected for the logical people group $(M=18.2 \%, S E=5.9 \%)$. For this reason, the decision was made to base the response time analysis on a different grouping variable that better reflected the influence of the ratio bias: a median split of the proportion of ratio bias responses. De Neys and Glumicic (2008) also used a median split method to analyze their response time data, after noting that a within-subjects comparison of optimal versus nonoptimal responses would be biased because it would exclude all the participants who made $100 \%$ optimal responses or 100\% nonoptimal responses (pp. 1268-1269). The median split resulted in a low-bias group (10 from the most people group and 15 from the logical people group) with $0 \%-12 \%$ ratio bias responses and a high-bias group (15 from the most people group and 10 from the logical people group) with $15 \%-96 \%$ ratio bias responses. Although the participants who received logical people instructions were more likely to be in the low-bias group than in the high-bias group ( $60 \%$ vs. $40 \%$, respectively), this difference was not significant.

A $2 \times(2 \times 3)$ repeated measures ANOVA was conducted on response time, as per the nonoptimal response analysis, but with instructions replaced by bias level (lowbias group or high-bias group), based on the median split outlined above. There were three significant effects: The main effects of bias level and direction are shown in Figure 1 and a two-way bias level $\times$ size interaction is shown in Figure 2. For bias level $[F(1,48)=14.756, p<.001]$, the high-bias group $(M=1,687 \mathrm{msec}, S E=149 \mathrm{msec})$ was faster than the low-bias group $(M=2,498 \mathrm{msec}$, $S E=149 \mathrm{msec})$. For direction $[F(1,48)=17.546, p<$ $.001]$, harmony trials $(M=1,881 \mathrm{msec}, S E=108 \mathrm{msec})$ were faster than conflict trials $(M=2,303 \mathrm{msec}, S E=$ $126 \mathrm{msec})$. For the bias level $\times$ size interaction $[F(2,47)=$ $5.050, p=.010]$, the low-bias group became slower as the ratio difference became smaller: Responses were significantly slower for ratios with a $1 \%-3 \%$ difference $(M=$ $2,648 \mathrm{msec}, S E=184 \mathrm{msec}$ ) than for ratios with a $7 \%-9 \%$ difference $(M=2,342 \mathrm{msec}, S E=145 \mathrm{msec})$. No such size effect was found for the high-bias group.

Correlations between the proportion of nonoptimal responses and response time were conducted separately for conflict and harmony trials. For conflict trials, the propor-

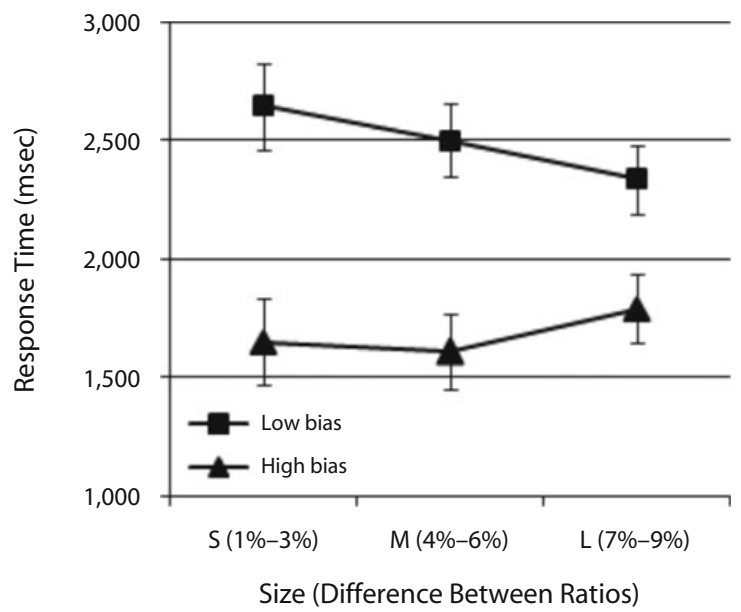

Figure 2. Interaction between bias level and size (small $>$ large for low-ratio-bias group), collapsed across direction. Bias level refers to a median split of participants based on the proportion of ratio bias responses they made (i.e., nonoptimal responses on conflict trials). Direction refers to which tray had the larger proportion of red jelly beans, the small tray (conflict trials) or the large tray (harmony trials). Size refers to the difference between the proportions of red jelly beans in the small and large trays on a given trial: small $(1 \%-3 \%)$, medium $(4 \%-6 \%)$, or large $(7 \%-9 \%)$. Error bars are one standard error of the mean. 
tion of nonoptimal responses (i.e., ratio bias responses) was negatively correlated with response time $(r=-.459$, $p=.001)$. For harmony trials, the correlation between nonoptimal responses (i.e., errors) and response time was not significant $(r=-.040, p>.1)$. These two correlation coefficients were significantly different from one another $(z=2.63, p<.05) .{ }^{2}$ Note that the range of nonoptimal responses in the harmony trials was $0 \%-58 \%$, so it is unlikely that the absence of a correlation was due to a floor effect on those trials.

\section{Discussion}

The results provide support for all three hypotheses. First, processing was shown to be slower when a different response was cued by assumed heuristic and analytic processing (e.g., 2/10 [analytic response] vs. 19/100 [heuristic response]) than when the same response was cued by heuristic and analytic processing (e.g., $2 / 10$ vs. $21 / 100$ [heuristic and analytic response]). This is consistent with the idea that people do experience a conflict when competing responses are cued and that conflict detection, or dealing with the consequences of that conflict, takes additional time to process. In addition, the direction and bias level factors did not interact. This indicates that even the highly biased participants took longer to respond to the conflict trials than to the harmony trials. This is crucial because it indicates that the highly biased reasoners were still sensitive to the presence of conflict between the two types of responses. Thus, the ratio bias phenomenon cannot be attributed to a mere failure to detect a conflict between the analytic and heuristically cued responses.

Second, the participants who relied more on the heuristic response (the high-bias group) were faster to respond than were those inclined to resolve the conflict in order to give the analytic response (the low-bias group). However, on the basis of this finding alone, it is possible that the apparent conflict resolution effect was simply driven by the participants who rushed through the task without considering the information properly; this would result in more nonoptimal responses and shorter response times across conflict and harmony trials, with no causal relation between the two. The correlation analysis therefore provides important support for the former interpretation: Only nonoptimal responding on conflict trials (i.e., ratio bias responses) was associated with shorter response times; nonoptimal responding on harmony trials (i.e., errors that were not consistent with heuristic or analytic processing) had no association with response time. Taken together, these findings are consistent with the idea that it takes additional time to resolve the conflict in order to give the analytic response.

Third, the amount of conflict did affect the response time for participants in the low-bias group. Specifically, latencies decreased as the difference between the ratios increased. This pattern supports the idea that it may be more difficult and/or more resources may be used to resolve the conflict in order to give the analytic response when the quantitative difference between response options is small. This could also explain why participants are gener- ally more likely to make a ratio bias response when the difference is small (cf. Denes-Raj \& Epstein, 1994).

The unexpected finding that the instructional manipulation (most people vs. logical people) did not determine the level of ratio bias use (high vs. low) in the median split is worth noting. Although the instructional manipulation did affect the mean proportion of ratio bias responses, individual differences in analytic processing appear to be a stronger determinant of ratio bias use for many participants. The written instructional manipulation may simply be too weak to affect responding in the hypothesized way, since the difference between the two groups was smaller than expected.

These results indicate that the response time findings of De Neys and colleagues (De Neys, 2006a, 2006b; De Neys \& Glumicic, 2008; De Neys et al., 2008) generalize to a different type of conflict task: one in which the heuristic response is cued by task-specific information rather than by prior beliefs, the analytic response is reached through a single identifiable rule, and the response times are on a much shorter time scale. Support was also found for an additional idea, that the amount of conflict may determine how easily the conflict can be resolved in order to give the analytic response.

\section{EXPERIMENT 2}

Having established that response time differences associated with conflict between heuristic and analytic responses can be found in the ratio bias task, the next aim was to manipulate the influence of the two responses and assess how this would affect processing time.

As was noted earlier, the impact of the heuristic response cue was enhanced by the use of a visual presentation (Rudski \& Volksdorf, 2002); the impact could, therefore, be reduced by replacing this aspect of the task with a simple verbal description of the ratio.

The ability to give analytic responses in conflict tasks has been associated with individual differences in working memory capacity (Stanovich \& West, 2000). Presumably, this is because analytic processing requires the use of working memory. Analytic processing can also be reduced by introducing a working memory load (WML), through the use of a secondary task (De Neys, 2006b).

\section{Aim and Hypotheses}

The aim of Experiment 2 was to test whether reducing the impact of the heuristic response and/or reducing the capacity to make the analytic response would change the response time effects found in Experiment 1.

It should be easier to resolve the conflict in order to give the analytic response when the heuristic cue is less compelling (i.e., a verbal rather than a visual representation of the ratios). A verbal format is therefore expected to decrease the proportion of nonoptimal responses and decrease the response time differences associated with conflict resolution (i.e., the low-bias group's taking longer to respond than the high-bias group and a significant correlation between ratio bias responding and response 
time). Processing times may also be longer in the verbal format, as compared with the visual format, due to additional reading time.

It should be harder to resolve the conflict in order to give the analytic response when available working memory capacity is reduced. A WML is therefore expected to increase the proportion of nonoptimal responses and increase the response time difference associated with conflict resolution (i.e., the low-bias group's taking longer to respond than the high-bias group). Processing times may also be longer, in general, when there is a WML.

\section{Method}

Participants. One hundred undergraduate students (55\% female, $45 \%$ male) with a mean age of 20.3 years $(S D=0.592)$ participated, in return for credit in a first-year psychology course at the University of New South Wales.

Design. The experiment was a $2 \times 2 \times(2 \times 3)$ design, based on format, WML, direction, and size. Format refers to how the ratios were represented: in pictures (as per Experiment 1) or words (a verbal description of the ratio). WML refers to whether there was a concurrent task (load group) or just the tray choice task (no-load group). The within-subjects variables were the same as those in Experiment 1: Direction refers to which tray had the higher percentage of red jelly beans, the small tray (conflict trials) or the large tray (harmony trials), and size refers to the percentage difference between ratios: $1 \%-3 \%$ (small), $4 \%-6 \%$ (medium), or $7 \%-9 \%$ (large).

Materials. The ratio pairs were based on the 54 conflict and harmony trials used in Experiment 1. For the word format, the jelly bean images within the tray picture were replaced by a verbal description of the ratio - for example, " 2 red jelly beans out of 10 total jelly beans." For the concurrent WML, a numerical rehearsal task (Waldron \& Ashby, 2001) was used. In this task, participants are briefly shown two single-digit numbers simultaneously, which differ in physical size (i.e., font size) and numerical value. After doing another task, they must then recall whether the left or the right number was larger in terms of either size or value. On $85 \%$ of the trials, the higher value is smaller in size. The task was chosen because it has been shown to impair performance on categorization tasks that are claimed to rely on the same kind of rule-based analytical processing that is claimed to underlie optimal responding in the ratio bias task (Waldron \& Ashby, 2001). The numerical nature of the secondary task overlaps with the numerical nature of the ratio bias task, thus perhaps enhancing the likelihood of interference.

Procedure. The participants completed the experiment on a computer. The instructions were the same as those in Experiment 1, except that all the participants received the most people version, because it resulted in more nonoptimal responses than did the logical people version (see Experiment 1 results).

The load group received additional instructions about how to do the numerical rehearsal task. They were told that they would be doing a memory task and a judgment task at the same time, which were not related but were of equal importance. When they clicked a button, the two numbers would appear for $200 \mathrm{msec}$; these were then masked for $200 \mathrm{msec}$ before the participants could continue to the tray choice task. After the tray choice, they were shown the word "size" or "value," and they had to click on a button to indicate whether the left or the right number was larger in terms of the given feature.

The tray choice task was set up the same way as in Experiment 1, except that the participants responded by clicking the left or right tray options with the mouse instead of pressing response keys. There was also an intertrial "continue" button to click, positioned so that the participants were required to move the mouse a short but consistent distance to make either response. These changes were made in order to keep the response mode (i.e., clicking a button with the mouse) consistent throughout the experiment for all the conditions and also to prevent the preemptive responding that affected some of the data in Experiment 1.

\section{Results}

Table 2 displays the mean proportion of nonoptimal responses and mean response time in each condition (across conflict and harmony trials). For the response time analysis, outliers $(>2.5 S D$ s from the mean) were replaced with the cutoff value. This was done separately for conflict and harmony trials for each participant and affected $4 \%$ of the data.

Nonoptimal choice. A $2 \times 2 \times(2 \times 3)$ repeated measures ANOVA was conducted on the proportion of nonoptimal responses, based on the variables of format (pictures or words), WML (load or no load), direction (conflict or harmony), and size (small, medium, or large). A nonoptimal response was defined as choosing the tray with the smaller percentage chance of winning. There were five significant effects: direction, size, and format main effects and direction $\times$ size and direction $\times$ format two-way interactions. For direction $[F(1,96)=27.159, p<.001]$, more nonoptimal responses were made on conflict trials $(M=27.9 \%, S E=3.3 \%)$ than on harmony trials $(M=$ $7.3 \%, S E=1.8 \%$ ), indicating a ratio bias effect. For size $[F(2,95)=16.804, p<.001]$, the proportion of nonoptimal responses decreased as the difference between the ratios increased, with a significant difference between small $(M=20.9 \%, S E=2.0 \%)$ and large $(M=14.4 \%, S E=$ $1.6 \%)$. The interaction with direction $[F(2,95)=3.195$, $p=.045]$ shows that this effect was greater on conflict trials than on harmony trials. For format $[F(1,96)=4.574$, $p=.035]$, the word format $(M=13.9 \%, S E=2.4 \%)$ resulted in significantly fewer nonoptimal responses than did the picture format $(M=21.3 \%, S E=2.4 \%)$. The interaction with direction $[F(1,96)=6.500, p=.012]$ shows that this effect was greater on conflict trials than on harmony trials.

There was no correlation between nonoptimal responding on the ratio bias task and performance on the WML task $(r=-.063, p=.535)$, despite high accuracy on the WML task $(M=90.3 \%, S D=11.2 \%)$.

Table 2

Mean Percentages of Nonoptimal Choices and Mean Response Times in Experiment 2

\begin{tabular}{|c|c|c|c|c|c|}
\hline & & \multicolumn{2}{|c|}{$\begin{array}{l}\text { Nonoptimal } \\
\text { Choice (\%) }\end{array}$} & \multicolumn{2}{|c|}{$\begin{array}{l}\text { Response } \\
\text { Time (msec) }\end{array}$} \\
\hline & & $M$ & $S E$ & $M$ & $S E$ \\
\hline Direction & $\begin{array}{l}\text { Conflict trials } \\
\text { Harmony trials }\end{array}$ & $\begin{array}{r}27.9 \\
7.3\end{array}$ & $\begin{array}{l}3.3 \\
1.8\end{array}$ & $\begin{array}{l}3,427 \\
2,751\end{array}$ & $\begin{array}{l}152 \\
142\end{array}$ \\
\hline Size & $\begin{array}{l}\text { Small } \\
\text { Medium } \\
\text { Large }\end{array}$ & $\begin{array}{l}20.9 \\
17.6 \\
14.4\end{array}$ & $\begin{array}{l}2.0 \\
1.8 \\
1.6\end{array}$ & $\begin{array}{l}3,007 \\
3,021 \\
3,239\end{array}$ & $\begin{array}{l}146 \\
157 \\
161\end{array}$ \\
\hline Format & $\begin{array}{l}\text { Picture } \\
\text { Word }\end{array}$ & $\begin{array}{l}21.3 \\
13.9\end{array}$ & $\begin{array}{l}2.4 \\
2.4\end{array}$ & $\begin{array}{l}2,870 \\
3,308\end{array}$ & $\begin{array}{l}197 \\
201\end{array}$ \\
\hline $\begin{array}{l}\text { Working } \\
\text { memory load }\end{array}$ & $\begin{array}{l}\text { No load } \\
\text { Load }\end{array}$ & $\begin{array}{l}18.1 \\
17.2\end{array}$ & $\begin{array}{l}2.4 \\
2.4\end{array}$ & $\begin{array}{l}3,264 \\
2,914\end{array}$ & $\begin{array}{l}194 \\
204\end{array}$ \\
\hline Bias level & $\begin{array}{l}\text { Low } \\
\text { High }\end{array}$ & $\begin{array}{r}6.0 \\
28.8\end{array}$ & $\begin{array}{l}1.8 \\
2.1\end{array}$ & $\begin{array}{l}3,408 \\
2,770\end{array}$ & $\begin{array}{l}199 \\
198\end{array}$ \\
\hline
\end{tabular}


Response time. As per the response time analysis in Experiment 1, the bias level variable was based on a median split of the proportion of ratio bias responses, resulting in a low-bias group $(n=49)$ with $0 \%-7 \%$ ratio bias responses and a high-bias group $(n=51)$ with $11 \%-100 \%$ ratio bias responses.

A $2 \times 2 \times 2 \times(2 \times 3)$ repeated measures ANOVA was conducted on response time, as per the nonoptimal response analysis, but with the additional factor of bias level (low-bias group or high-bias group). There were five significant effects: bias level and direction main effects, bias level $\times$ direction and WML $\times$ direction two-way interactions, and a bias level $\times$ direction $\times$ WML threeway interaction. The main effects and the two-way interaction between them are illustrated in Figure 3. For bias level $[F(1,92)=5.142, p=.026]$, the high-bias group $(M=2,770 \mathrm{msec}, S E=198 \mathrm{msec})$ was faster than the low-bias group $(M=3,408 \mathrm{msec}, S E=199 \mathrm{msec})$. For direction $[F(1,92)=58.687, p<.001]$, responses on the harmony trials $(M=2,751 \mathrm{msec}, S E=142 \mathrm{msec})$ were faster than those on the conflict trials $(M=3,427 \mathrm{msec}$, $S E=152 \mathrm{msec})$. For the bias level $\times$ direction interaction $[F(1,92)=6.312, p=.014]$, the difference between conflict and harmony trials was larger for the high-bias group than for the low-bias group. For the WML $\times$ direction interaction $[F(1,92)=5.012, p=.028]$, the difference between conflict and harmony trials was larger for the noload group than for the load group. For the bias level $\times$ direction $\times \mathrm{WML}$ interaction $[F(1,92)=7.828, p=.006]$,

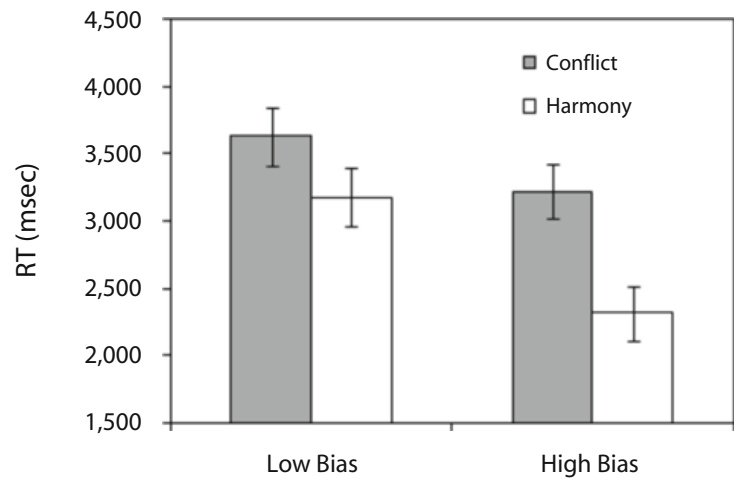

Bias Level

Figure 3. Main effects of bias level (low $>$ high) and direction (conflict $>$ harmony) and the two-way interaction between them (conflict - harmony difference greater for the high-ratio-bias group), collapsed across size, format, and working memory load. Bias level refers to a median split of participants based on the proportion of ratio bias responses they made (i.e., nonoptimal responses on conflict trials). Direction refers to which tray had the larger proportion of red jelly beans, the small tray (conflict trials) or the large tray (harmony trials). Size refers to the difference between the proportions of red jelly beans in the small and large trays on a given trial: small $(1 \%-3 \%)$, medium $(4 \%-6 \%)$, or large $(7 \%-9 \%)$. Format was either visual (pictures of trays) or verbal (description of trays), and working memory load was either present (concurrent secondary task) or absent. Error bars are one standard error of the mean. the greater conflict-harmony difference in the high-bias group was found only for those with no WML.

Correlations between the proportion of nonoptimal responses and response time were conducted separately for conflict and harmony trials. For conflict trials, the proportion of nonoptimal responses (i.e., ratio bias responses) was negatively correlated with response time $(r=-.272$, $p=.006)$. For harmony trials, there was no significant correlation between nonoptimal responses (i.e., errors) and response time $(r=.121, p=.232)$. These correlations differed significantly from each other $(z=2.26, p<$ $.05)$. Note that the range of nonoptimal responses in the harmony trials was $0 \%$ to $100 \%$, so it is unlikely that the absence of a correlation was due to a floor effect on those trials.

\section{Discussion}

The basic pattern of effects found in Experiment 1 was replicated in Experiment 2. There were more nonoptimal responses on conflict trials (e.g., 2/10 vs. 19/100) than on harmony trials (e.g., $2 / 10$ vs. $21 / 100$ ), and processing was slower on conflict trials than on harmony trials. Interestingly, direction and bias level did interact in Experiment 2, but as Figure 3 shows, the interaction was driven by the increased difference between conflict and harmony trials for the high-bias group. Thus, consistent with Experiment 1, the ratio bias cannot be attributed to a failure to detect the conflict in this group.

The tendency to choose nonoptimally was reduced when ratios were presented in verbal format, as compared with visual format. This result lends some support to the notion that the heuristic response is the product of simple associative processing, driven by the salience of the external heuristic cue. However, format did not have a significant effect on response time. The participants who relied more on the heuristic response (the high-bias group) were faster to respond than were those who were more inclined to resolve the conflict in order to give an analytic response (the low-bias group); and nonoptimal responses on conflict trials (i.e., ratio bias responses), but not on harmony trials (i.e., errors), were correlated with shorter response times.

Although the amount of conflict (e.g., a 1\% vs. 9\% difference between ratios) was found to influence response time for the low-bias group in Experiment 1, this effect was not replicated in Experiment 2. Reducing the participants' capacity to make the analytic response through the addition of a WML had none of the predicted effects on nonoptimal responding or response time. This was surprising, given De Neys's (2006b) finding that a different type of concurrent task increased heuristic responding in base rate neglect problems. The absence of an impact may be due to the repetitive nature of the trials and the simplicity of the rules required for analytic processing (relative to the base rate neglect problems), which allowed the participants to concentrate on both tasks equally. This idea is supported by the lack of correlation between performance on the two tasks. Alternatively, it could indicate that optimal and nonoptimal responding in the ratio bias task are based on a single processing system and, so, were equally 
(un)affected by the WML. This idea is discussed further in the General Discussion section. Finally, the unexpected interactions involving bias level (low or high), direction (conflict or harmony), and WML (no load or load) can be attributed to a greater conflict detection effect (conflict $>$ harmony) for the high-bias, no-load group, as compared with all the other groups. It is unclear why this particular group differed, since the interactions are not related to any of the hypothesized effects.

\section{GENERAL DISCUSSION}

The popularity of dual-process models sometimes belies complex issues regarding how the proposed processes compete or exchange information (Newell, 2009). The present research builds on promising work, initiated in a discussion article by Evans (2007), on how the perceived conflict between the outputs of proposed processes might be detected and resolved. A simple ratio bias task was used as a vehicle for investigating conflict between heuristic responding (choose the tray with the higher numerator) and analytic responding (consider the entire ratio and choose the tray with the higher percentage). The research extended previous work on the ratio bias task by measuring response times across multiple trials to provide more insight into the mechanisms underlying the two types of responding.

The results of both experiments provide quantitative evidence for the observation that participants experience a conflict (Denes-Raj \& Epstein, 1994) in the ratio bias task when the heuristic response and the analytic response suggest different answers. Across both experiments, the participants made more nonoptimal choices on conflict trials and took longer to make those responses. Furthermore, in Experiment 2, the latter effect was magnified for those participants who displayed a high level of biased responding, suggesting that biased responding is not simply the result of a failure to detect the conflict. The fact that the response time differences between conflict and harmony trials were found in two separate experiments, across manipulations of instruction, presentation format, and concurrent WML, illustrates the robustness of these effects.

The use of a simpler conflict task allowed processing differences to be demonstrated on a much shorter time scale (within a 2-sec range), which may more closely reflect the automatic heuristic processing defined in dualprocess models. The results also support the assumption that optimal responding is the product of analytic rulebased processing and nonoptimal responding is the product of heuristic cue-based processing in the ratio bias task. Support for these suppositions comes from the increase in optimal responding as a function of instructions emphasizing logical responding (Experiment 1) and an increase in nonoptimal responding with visual, as opposed to verbal, presentation (Experiment 2).

\section{Dual Processes: Intervention or Parallel Operation?}

Evans (2007) proposed two possibilities for the way in which dual processes might interact. The default- interventionist (DI) model proposes a serial framework in which heuristic processes (and thus, responses) are the default and these are intervened upon, later in processing, by analytic processes only when some conflict is detected; thus, monitoring is somewhat lax (De Neys \& Glumicic, 2008). In contrast, parallel models (e.g., Denes-Raj \& Epstein, 1994; Sloman, 1996) suggest that heuristic and analytic processes occur simultaneously, leading to constant and effective monitoring and the experience of conflict.

The present data suggest that participants are very good at detecting conflict in the ratio bias paradigm. Even those participants who tended to make more heuristic responses (high-bias group) had longer average latencies on conflict trials than on harmony trials, indicating the detection (or resolving the consequences) of conflict. These results appear to be consistent with the effective monitoring proposed by the parallel model. The DI model would, arguably, predict that participants in the high-bias group should be less likely to detect conflict; if a participant makes the heuristic response repeatedly over a number of trials, this implies that heuristic processing is dominating, leaving little opportunity for analytic processes to note a conflict and intervene. The present data thus support earlier accounts of performance in the ratio bias paradigm, which have also been interpreted in the context of parallel models (e.g., CEST; Denes-Raj \& Epstein, 1994; Epstein \& Pacini, 2000-2001; Kirkpatrick \& Epstein, 1992).

However, as De Neys and Glumicic (2008) correctly pointed out, neither an extreme serial DI model nor a pure parallel model make sense once their underlying assumptions are scrutinized. In the DI model, some analytic processing must occur all the time for a conflict to be detected. It makes no sense to say that analytic processes will be engaged only when a conflict is detected. How could conflict be detected if at least minimal analytic monitoring was not operating? For the parallel model, the principle of cognitive economy suggests that analytic processes would be redundant in those instances in which both heuristic and analytic routes lead to the same answer (e.g., harmony trials in the present context). Why would a system "bother with" the slower, more capacity-intensive process when an "easy" heuristic solution presents itself?

De Neys and Glumicic (2008) resolved the perceived inadequacies of these "pure" accounts by invoking a "hybrid two-stage model" characterized by a "shallow analytic monitoring process" and an "optional deeper processing stage" to accompany the ever-present heuristic processing (p. 1278). The basic idea is a model in which shallow analytic processing is always engaged in order for conflict to be detected and deeper analytic processing is sanctioned only once there is a conflict between the shallow processing and the heuristic response. Such a hybrid model would fit the present data. The shallow analytic processing is always engaged - especially likely given the within-subjects manipulation of conflict and harmony trials - but the deeper, more time-consuming analytic processing is invoked only when a detected conflict needs to be resolved (i.e., conflict trials). Thus, the longer response times on conflict trials are due to the attempt to resolve the conflict, arguably via inhibition of the heuristic response. ${ }^{3}$ 
The results show that the success of this conflict resolution is dependent on the probability difference between ratios, the format of the information (verbal or visual), and the emphasis of the instructions (a logical people or most people perspective).

\section{Is a Dual-Process Account Necessary?}

Although the two-stage hybrid model of De Neys and Glumicic (2008) could account for the data, is it necessary to invoke a dual-process explanation? Dual-process models are extremely popular, but popularity does not mean that they are correct, nor does it necessarily imply that they are useful for theoretical progress (cf. Mitchell et al., 2009). The preceding interpretation and framing is entirely in terms of the competition between the outputs of two distinct types of processing. Even if one is agnostic with respect to whether these processes are themselves encompassed by distinct systems, the very presence of duality necessitates complex accounts of how the two entities (or products thereof) interact. But is such theorizing beneficial?

It appears that even when duality is proposed, there is little consensus across different areas of research on how such interaction might occur. For example, an influential dual-system model of category learning, COVIS (Ashby, Alfonso-Reese, Turken, \& Waldron, 1998), proposes an initial bias toward an explicit hypothesis-testing system, which is then usurped by an automatic system when the explicit system fails to learn. In contrast, the models discussed here suggest that the initial bias is toward the automatic system, which is corrected by the explicit/analytic system only when conflicts are detected. Even authors strongly aligned with dual-process models caution that "generic dual-system theory is over-simplified and misleading" (Evans, 2008, p. 270).

Rather than forcing data to fit with a competing systems account, an attempt should be made (although it rarely is) to examine the capability of single-system accounts (cf. Hammond, 1996; Newell \& Dunn, 2008; Osman, 2004). The essential features of the present data to be explained are the increase in response times on conflict, relative to harmony, trials and the longer response times for participants who give more optimal responses. Hammond's cognitive continuum theory (CCT) provides an alternative way of viewing these results, on the basis of the idea that task features determine placement on a cognitive continuum - from fast intuitive thinking to slower analytic thinking.

According to CCT, the effects attributed to conflict detection (i.e., longer response times on conflict trials) and conflict resolution (i.e., analytic responses associated with longer response times) would simply be a result of task feature differences. Conflict trials could be viewed as inherently more analysis inducing than harmony trials, because on conflict trials two justifiable responses can be readily seen (the tray with the higher number or the tray with the higher percentage). To explain the conflict detection effect, this difference would prompt a shift in processing toward the analytic end of the continuum, which would slow processing on conflict, relative to harmony, trials. The conflict resolution effect can be explained in a similar way. If the availability of two justifiable responses on conflict trials results in placement toward the analytic end of the continuum, participants will (1) give the optimal response and (2) have a longer processing time, but these two effects are not causally related; this is a notable difference between a CCT interpretation and the idea that successful conflict resolution is required to give the optimal response. The dual-process explanation is that the extra step of resolving the conflict that arises from the competing responses of two processes directly adds to the processing time, causing longer response times for those who complete this more often. However, the necessary analysis method used in both the present and past research cannot establish such a causal link, only a correlation between more optimal responses and longer response times. In contrast, CCT predicts that there is a correlation but no causal link, for which the present data would be sufficient evidence.

One could go a step further in suggesting that even the continuum of analytic/heuristic processing is not required. The increased response times for conflict, relative to harmony, trials could be attributed to the fact that two possible answers are apparent on conflict trials but only one is apparent on harmony trials. The availability of two possible answers could simply require more (but not different) processing, resulting in longer response times. Similarly, the increase in response times for those who give more optimal responses could be the result of the fact that people who thought about (processed) the problem for a longer time were more likely to decide on the higher percentage (cf. Evans's [2007] discussion of the quality vs. quantity of processing). In fact, the failure to find a significant effect of memory load in Experiment 2 supports the notion that the processes underlying optimal and nonoptimal responding are not qualitatively different, especially since proponents of dual-process models identify disruption by WML as a key indicator of the involvement of System 2/ analytic processes (Evans, 2008).

As was noted in the introduction, the aim of these experiments was to investigate whether processing effects found in past research could be generalized to a simpler and faster conflict task, rather than to provide a definitive test between dual- and single-process accounts. The more modest aim of the discussion above is simply to highlight the fact that constraining data interpretation to fit accounts involving two opposing forms of processing may not always be beneficial. For the present results, a hybrid two-stage model in the spirit of dual-process theories can explain the data, but arguably, a single-process model can fare equally well. The account one favors might just come down to theoretical predilection.

\section{AUTHOR NOTE}

The support of the Australian Research Council (DP 0877510 , awarded to the second author) is gratefully acknowledged. We thank Wim De Neys and Jonathan Evans for extremely useful comments on an earlier draft of the manuscript. Correspondence concerning this article should be addressed to B. R. Newell, School of Psychology, University of New South Wales, Sydney 2052, NSW, Australia (e-mail: ben .newell@unsw.edu.au). 


\section{REFERENCES}

Alonso, A., \& Fernández-Berrocal, P. (2003). Irrational decisions: Attending to numbers rather than ratios. Personality \& Individual Differences, 35, 1537-1547.

Ashby, F. G., Alfonso-Reese, L. A., Turken, A. U., \& Waldron, E. M. (1998). A neuropsychological theory of multiple systems in category learning. Psychological Review, 105, 442-481.

Bonner, C., \& Newell, B. R. (2008). How to make a risk seem riskier: The ratio bias versus construal level theory. Judgment \& Decision Making, 3, 411-416.

Dale, D., Rudski, J., Schwarz, A., \& Smith, E. (2007). Innumeracy and incentives: A ratio bias experiment. Judgment \& Decision Making, 2, 243-250.

Denes-RaJ, V., \& Epstein, S. (1994). Conflict between intuitive and rational processing: When people behave against their better judgment. Journal of Personality \& Social Psychology, 66, 819-829.

De Neys, W. (2006a). Automatic-heuristic and executive-analytic processing in reasoning: Chronometric and dual task considerations Quarterly Journal of Experimental Psychology, 59, 1070-1100.

De Neys, W. (2006b). Dual processing in reasoning: Two systems but one reasoner. Psychological Science, 17, 428-433.

De Neys, W., \& Franssens, S. (2009). Belief inhibition during thinking: Not always winning but at least taking part. Cognition, 113, 45-61. doi:10.1016/j.cognition.2009.07.009

De Neys, W., \& Glumicic, T. (2008). Conflict monitoring in dual process theories of thinking. Cognition, 106, 1248-1299.

De Neys, W., Vartanian, O., \& Goel, V. (2008). Smarter than we think: When our brains detect that we are biased. Psychological Science, 19, 483-489.

EPSTEIN, S., \& PACINI, R. (2000-2001). The influence of visualization on intuitive and analytical information processing. Imagination, Cognition \& Personality, 20, 195-216.

Evans, J. ST. B. T. (2006). The heuristic-analytic theory of reasoning Extension and evaluation. Psychonomic Bulletin \& Review, 13, 378 395

Evans, J. St. B. T. (2007). On the resolution of conflict in dual process theories of reasoning. Thinking \& Reasoning, 13, 321-339.

Evans, J. St. B. T. (2008). Dual-processing accounts of reasoning, judgment, and social cognition. Annual Review of Psychology, 59, 255 278 .

Franssens, S., \& DE Neys, W. (2009). The effortless nature of conflict detection during thinking. Thinking \& Reasoning, 15, 105-128.

Gigerenzer, G., Hell, W., \& Blank, H. (1988). Presentation and content: The use of base rates as a continuous variable. Journal of Experimental Psychology: Human Perception \& Performance, 14, 513-525.

Goldstein, D. G., \& Gigerenzer, G. (2002). Models of ecological rationality: The recognition heuristic. Psychological Review, 109, 7590.

HAMmond, K. R. (1996). Human judgment and social policy. New York: Oxford University Press.

Howell, D. C. (2002). Statistical methods for psychology (5th ed.). Pacific Grove, CA: Thomson Learning.

Kahneman, D., \& Frederick, S. (2002). Representativeness revisited: Attribute substitution in intuitive judgment. In T. Gilovich, D. Griffin, \& D. Kahneman (Eds.), Heuristics and biases: The psychology of intuitive judgment (pp. 49-81). Cambridge: Cambridge University Press.

Kahneman, D., \& Tversky, A. (1973). On the psychology of prediction. Psychological Review, 80, 237-251.
KirkPAtrick, L., \& EPSTEIn, S. (1992). Cognitive-experiential selftheory and subjective probability: Further evidence for two conceptual systems. Journal of Personality \& Social Psychology, 63, 534-544.

Mitchell, C. J., DeHouwer, J., \& Lovibond, P. F. (2009). The propositional nature of human associative learning. Behavioral \& Brain Sciences, 32, 183-198.

Newell, B. R. (2009). What is the link between propositions and memories? Behavioral \& Brain Sciences, 32, 219.

Newell, B. R., \& Dunn, J. C. (2008). Dimensions in data: Testing psychological models using state-trace analysis. Trends in Cognitive Sciences, 12, 285-290.

OSMAN, M. (2004). An evaluation of dual-process theories of reasoning. Psychonomic Bulletin \& Review, 11, 988-1010.

Pinto-Prades, J. L., Martinez-Perez, J. E., \& Abellán-Perpiñán, J. M. (2006). The influence of the ratio bias phenomenon on the elicitation of health states utilities. Judgment \& Decision Making, 1, 118133

RUdSKI, J. M., \& VolKsdorf, J. (2002). Pictorial versus textual information and the ratio-bias effect. Perceptual \& Motor Skills, 95, 547554

Sloman, S. A. (1996). The empirical case for two systems of reasoning. Psychological Bulletin, 119, 3-22.

Sloman, S. A., Over, D., Slovak, L., \& Stibel, J. M. (2003). Frequency illusions and other fallacies. Organizational Behavior \& Human Decision Processes, 91, 296-309.

Stanovich, K. E., \& West, R. F. (1998). Cognitive ability and variation in selection task performance. Thinking \& Reasoning, 4, 193-230.

Stanovich, K. E., \& WeSt, R. F. (2000). Individual differences in reasoning: Implications for the rationality debate. Behavioral \& Brain Sciences, 23, 645-726.

WALDRon, E. M., \& Ashby, F. G. (2001). The effects of concurrent task interference on category learning: Evidence for multiple category learning systems. Psychonomic Bulletin \& Review, 8, 168-176.

Wilson, T. D., \& Schooler, J. W. (1991). Thinking too much: Introspection can reduce the quality of preferences and decisions. Journal of Personality \& Social Psychology, 60, 181-192.

YAMAGISHI, K. (1997). When a $12.86 \%$ mortality is more dangerous than $24.14 \%$ : Implications for risk communication. Applied Cognitive Psychology, 11, 495-506.

\section{NOTES}

1. When formulating our predictions and hypotheses, for the sake of consistency (especially with the work of De Neys and colleagues), we use the terms analytic, heuristic, and conflict in the same manner as in the dual-process literature. However, in the General Discussion section, we note that these concepts do not have to be invoked to explain the reported data.

2. The correlation coefficients in Experiments 1 and 2 were compared by transforming each $r$ to $r^{\prime}$, using the Fisher transformation and then using these transformed values to calculate the $z$ statistic (Howell, 2002).

3. Note that although the present data cannot confirm whether or not this is the mechanism used to resolve the conflict, see De Neys and Franssens (2009) for evidence of inhibition in other conflict tasks.

(Manuscript received January 30, 2009 revision accepted for publication September 8, 2009.) 